\title{
Studies on Single Turn Extraction for a Superconducting Cyclotron
}

\author{
J.-W. Kim, A. Goto, T. Mitsumoto, and Y. Yano \\ Cyclotron Lab., RIKEN, Japan
}

\section{Abstract}

Feasibility of single turn extraction in a high-field separated sector cyclotron has been accessed. Three key requirements are: 1) larger last-turn separation than the beam width, 2) preservation of beam quality throughout the acceleration, and 3) phase space matching at injection. To enlarge separation of the last-turn orbital precession will be induced at the injection. Traversal of the $\nu_{r}=1.5$ resonance can affect the radial beam width of light nuclei accelerating to the energy above $300 \mathrm{MeV} / \mathrm{u}$. Tolerance to the gradient field of third harmonic, which causes the half-integer resonance in lowest order, was estimated to be 0.1 gauss $/ \mathrm{cm}$. The effects of vertical resonances were also evaluated, and the result was used in the yoke design. Moreover, longitudinal space charge forces were computed for a realistic charge distribution of beam on the estimation of the limiting current.

\section{INTRODUCTION}

A superconducting separated-sector cyclotron is to be built at RIKEN as the final stage energy-booster of the primary beam in the Radio Isotope Beam Factory (RIBF) [1]. A prototype sector magnet which is a $1 / 1$ scale for the complete design verification, is scheduled to be complete this year [2]. The prototype contains a part of superconducting trim coils and a superconducting injection channel magnet to test the major cyclotron components under actual magnetic fields. The maximum fields in the extraction region of the sector magnet are plotted in Fig. 1 for some design particles.

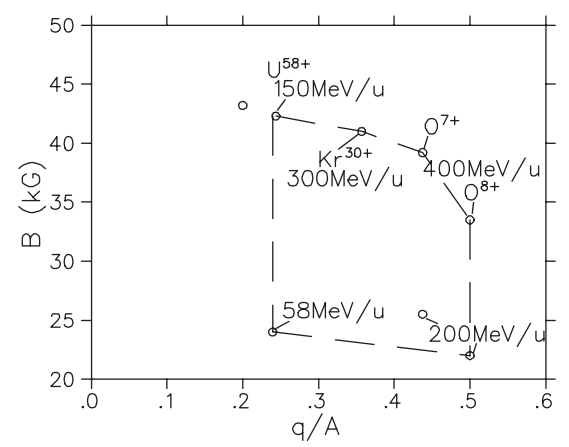

Figure 1: Maximum magnetic fields in the extraction region of the SRC for design nuclei.

To lessen beam induced damages extraction should be carried out without much losses. A larger last-turn separation than the radial beam spread and a preservation of beam quality are the prerequisites for single turn extraction. Orbit trackings have been performed for design nuclei using isochronized magnetic fields which were obtained by leastsquare fittings with the main and trim coil fields calculated using TOSCA [3] at several different levels of excitations. The tune diagram of those nuclei is shown in Fig. 2.

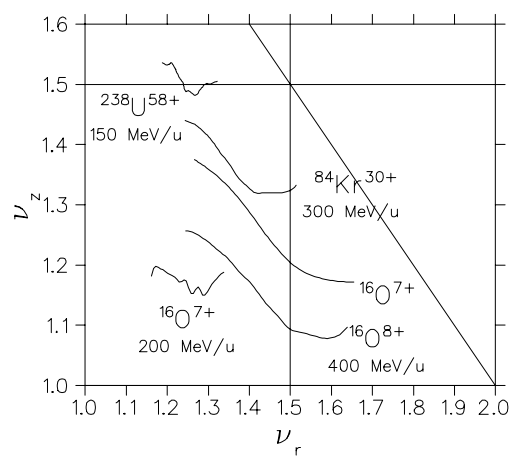

Figure 2: Tune diagram of the design nuclei in the SRC.

The six dimensional phase spaces of beam should be well matched with acceptance of each accelerator to avoid deterioration of beam qualities. The accelerator chain in the RIBF is shown in Fig. 3. We are particularly concerned with matching at the SRC. The transverse phase spaces need additional selection prior to the injection into the SRC. The design of the injection line is underway, and will be described elsewhere.

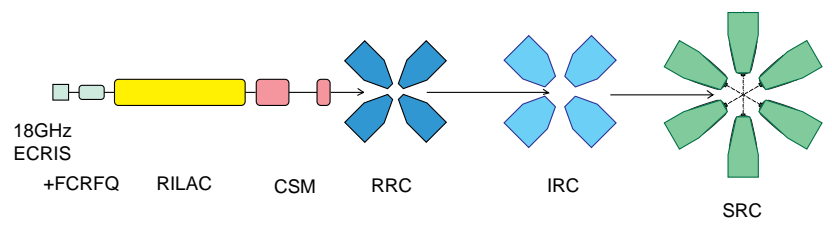

Figure 3: Accelerator chain of the primary beam in the RIBF.

\section{ENHANCEMENT OF LAST-TURN SEPARATION}

The radius-gain of a beam in cyclotron is given as below

$$
\frac{\Delta r}{d n}=r \frac{\gamma}{\gamma+1} \frac{\Delta E}{E} \frac{1}{\nu_{r}^{2}},
$$

where $\gamma$ is the relativistic factor, $E$ is the kinetic energy of beam, and $\Delta E$ is the energy gain per turn. The main design particle is a light ion accelerating to the highest design energy $400 \mathrm{MeV} / \mathrm{u}$ because of its smallest turn separation. The radius gain of such an ion at the extraction is about 2$3 \mathrm{~mm}$ when a practical energy gain by rf cavities is used, 
while the radial beam spread is about $4 \mathrm{~mm}$ when the beam emittance is $3.5 \pi \mathrm{mm} \cdot \mathrm{mrad}$. Hence, separation of the lastturn needs to be enlarged.

The major parameters which determine the turn separation are the energy gain and $\nu_{r}$. In the initial design three cavities, which were symmetrically interspersed among six sectors, were chosen to be used because three unoccupied valleys can comfortably accommodate the flat-top cavity and electrostatic injection and extraction elements. But it was conceived afterward to be worthwhile to employ four cavities at the cost of the simple layout. Figure 4 shows the layouts of both schemes. The adoption of four cavities makes the beam injection more intricate and the placement of the components more congested, but benefits the limiting current by space charge forces. On the other hand, the location of the extraction radius is to be finalized in relation to the $\nu_{r}$ value, while maintaining the necessary field isochronism.

The main cavity is of a single-gap type, the maximum voltage is $600 \mathrm{kV}$ in the extraction region at the power loss of $90 \mathrm{~kW}$, and the voltage of the flat-top cavity is $200 \mathrm{kV}$ at $30 \mathrm{~kW}$ [4].

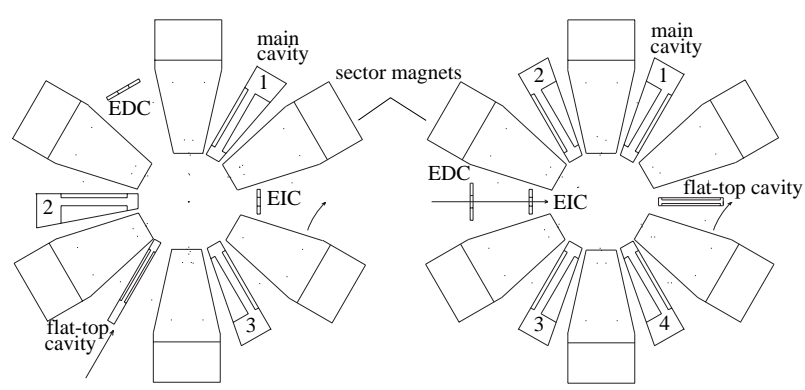

Figure 4: The SRC layouts with three or four cavities. Injection and extraction routes are indicated by arrows.

The last-turn separation is to be enhanced by offcentering at the injection. In usual compact cyclotrons, the orbital off-centering at the traverse of the radial resonance $\nu_{r}=1$ would hurt beam qualities, but in high energy separated-sector cyclotrons the orbit can be off-centered from the point of injection. The orbit separation with precession can be written as [5]

$$
\Delta r_{p}=\Delta r_{c}+A \sin \left(2 \pi \nu_{r}\right),
$$

where $\Delta r_{c}$ is the radius-gain of a centered beam, which is the same as $\Delta r$ of Eq. 1, and $A$ is the amplitude of precession. Figure 5 shows the radius-gain as a function of energy gain when the $\mathrm{O}^{7+}$ beam is centered and off-centered. In fact, the radius-gain by three cavities may be sufficient to achieve the extraction efficiency over $99 \%$ for the beam with current of $1 \mathrm{p} \mu \mathrm{A}$ aimed at Phase I of the project [1] insofar as the beam quality is maintained. However, the four-cavity scheme is largely beneficial on the high current operation as demonstrated at the PSI proton cyclotron [6].

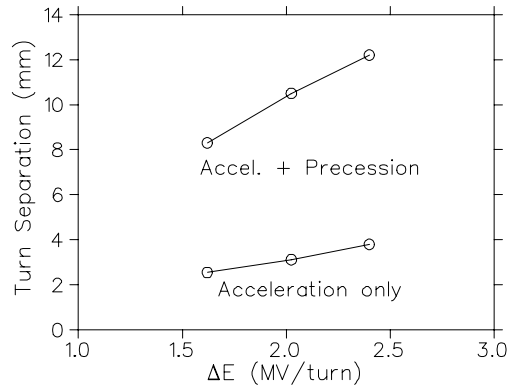

Figure 5: Last-turn separation of the $400 \mathrm{MeV} / \mathrm{u} \mathrm{O}^{7+}$ beam when the beam is centered and off-centered at injection.

\section{PRESERVATION OF BEAM QUALITY}

\subsection{Effects of resonances}

Resonance traversal and approach, which affect beam qualities, have been investigated by workers for separatedsector cyclotrons [7]. We have performed similar studies using numerical methods for the SRC which exhibits a larger flutter field and thus stronger vertical focusing force than in existent separated-sector cyclotrons.

When the SRC accelerates light nuclei above $300 \mathrm{MeV} / \mathrm{u}$, the $\nu_{r}=3 / 2$ resonance is traversed. In the presence of the gradient field of third harmonic, the radial beam spread will increase above the resonance due to mismatching in eigen-ellipses across the resonance. Tolerance has been estimated, and is currently set to be 0.1 gauss $/ \mathrm{cm}$. Figure 6 shows a case of rather significant mismatch by the imposed gradient field of 0.7 gauss $/ \mathrm{cm}$. Orbital precession would then become less effective in enhancing separation of the last-turn.

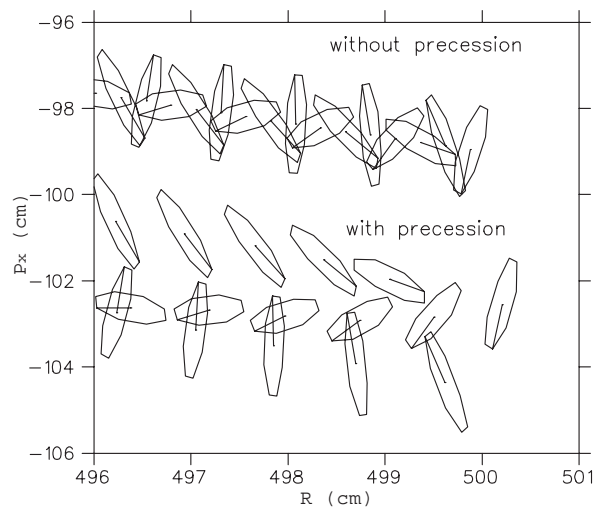

Figure 6: Phase space motion with and without precession near the extraction region. The third harmonic gradient field of 0.7 gauss $/ \mathrm{cm}$ is imposed.

As shown on the tune diagram in Fig. 2, the $\nu_{z}=3 / 2$ is traversed while the $\nu_{z}=1$ is kept away. This tune arrangement is due to the fact that the integer imperfection resonance is more harmful than the half-integer one. The $\nu_{z}$ of the ions with $\mathrm{q} / \mathrm{A}=0.5$ is 1.07 under the design field at the energy around $390 \mathrm{MeV} / \mathrm{u}$, and is 1.08 at $400 \mathrm{MeV} / \mathrm{u}$. The first 
harmonic $B_{r}$ near the $\nu_{z}=1$, its presence being everywhere in the cyclotron, induces a vertically coherent oscillation whose amplitude $\left(z_{e o}\right)$ is given if the smooth approximation is used by

$$
z_{e o}(\omega t)=r \frac{<B_{r}>}{<B_{z}>} \frac{1}{\nu_{z}^{2}-1} \cos (\omega t+\phi)
$$

where the bracket indicates an azimuthal average. Considering that the half height of the beam chamber is $3 \mathrm{~cm}$, the first harmonic $B_{r}$ should be kept much smaller than 10 gauss if the minimum $\nu_{z}$ is 1.07 .

\subsection{Space charge effects}

Space charge forces, transversal and longitudinal, limit the beam current in a cyclotron [8]. The transversal space charge forces are usually crucial at the low energy end of the cyclotron chain, thus at the injection into the RRC, while the longitudinal space charge forces can limit the beam current throughout the chain. The transversal limiting current at the $\mathrm{RRC}$ is high in the range of tens of $\mathrm{mA}$. Hence, the effects of longitudinal space charge forces have been mainly considered.

To compute the longitudinal space charge forces, the beam charge distribution should be known, but the actual charge distribution can be complicated especially when the orbits are in precession as seen in Fig. 6. The distribution has been measured for the Ti beam extracted from the RRC [9] as shown in Fig. 7. At the injection point of the SRC the charge distribution was obtained by ion tracking through the transport line which involves a buncher/debuncher for longitudinal phase space matching. The buncher is to be located in between the RRC and IRC.

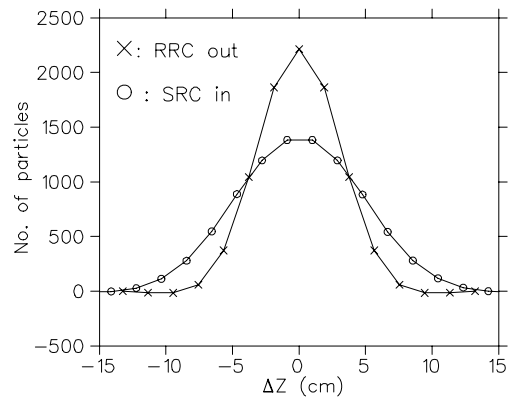

Figure 7: Charge distribution of beam extracted from the RRC as a function of bunch length, which is measured, and at the injection point of the SRC.

The Gordon formalism [10] was used to compute the electric fields of beam bunch as a function of azimuth. The formalism includes image charge effects due to the beam chamber. The electric field as a function of $\phi$ is given as

$$
E_{\theta}(\phi)=2 F_{0} G(\phi) \gamma^{3}
$$

where $F_{0}$ is the quantity having an electric field unit and $\mathrm{G}(\phi)$ is a dimensionless field function specified by the geometric parameters. $G(\phi)$ was calculated for the SRC beam with charge distribution of Fig. 7, and is plotted in Fig. 8. The beam height is assumed to be $6 \mathrm{~mm}$ with a rectangular charge distribution. Conducting planes are at $\mathrm{z}= \pm 3 \mathrm{~cm}$.

The limiting current is defined as the current when the last-turn separation disappears due to the beam energy spreads induced by longitudinal space charge forces. The maximum electric field at $1 \mathrm{p} \mu \mathrm{A}$ is about $25 \mathrm{~V} / \mathrm{m}$ for the $\mathrm{O}^{7+}$ beam, and the limiting current is estimated to be 3 $\mathrm{p} \mu \mathrm{A}$.

The dashed line in Fig. 8 indicates a correction of the space charge forces by controlling the relative rf phase between the main and flat-top cavities. If the charge distribution of actual beam has symmetry with respect to the central phase, such a correction is expected.

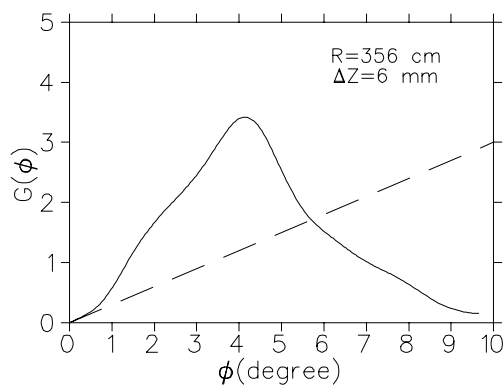

Figure 8: G( $\phi)$ as a function of phase angle assuming symmetric beam charge distribution w.r.t. the central phase. The dashed-line indicates a compensation of maximum $\mathrm{E}_{\theta}$.

\section{CONCLUDING REMARKS}

The number of rf cavity was increased from 3 to 4 for further enhancement of the last-turn separation at the cost of comfortable layout of the cyclotron components. The prototype magnet which is under construction will be used to gain experiences in alignments and thus to better estimate unwanted harmonic field components, as well as to test the performance of the sector-magnet. The longitudinal space charge forces have been approximately computed, so that continual efforts are required along with measurements.

\section{REFERENCES}

[1] Y. Yano et al., Proc. of 1997 Part. Accel. Conf., 930 (1997).

[2] T. Kawaguchi et al., Proc. of 15th Int. Conf. of Cyclotron and Their Applications, in press (1998).

[3] TOSCA is a product of Vector Fields, Oxford, England.

[4] N. Sakamoto et al., Proc. of 15th Int. Conf. of Cyclotron and Their Applications, in press (1998).

[5] W.M Schulte et al., IEEE Trans. on Nucl. Sci., NS-26, 2392 (1979).

[6] Th. Stammbach et al., NIM B113, 1 (1996).

[7] M.M. Gordon, Annals of Phys. 50, 571 (1968).

[8] W. Joho, Proc. of 9th Int. Cyclotron Conf., 337 (1981).

[9] H. Kumagai, Internal note, Linac Lab., RIKEN, (1998).

[10] M.M. Gordon, Proc. of 5th Cyclotron Conf., 305 (1969). 